\title{
Search and Congestion in Complex Networks
}

\author{
Alex Arenas ${ }^{1}$, Antonio Cabrales ${ }^{2}$, Albert Díaz-Guilera ${ }^{3}$, Roger Guimerà ${ }^{4}$, and \\ Fernando Vega-Redondo ${ }^{5}$ \\ 1 Departament d'Enginyeria Informàtica, Universitat Rovira i Virgili, Tarragona \\ 2 Departament d'Economia i Empresa, Universitat Pompeu Fabra, Barcelona \\ 3 Departament de Física Fonamental, Universitat de Barcelona, Barcelona \\ 4 Departament d'Enginyeria Química, Universitat Rovira i Virgili, Tarragona \\ 5 Departament de Fonaments d'Anàlisi Econòmica, Universitat d'Alacant, Alacant
}

\section{Introduction}

In recent years, the study of static and dynamical properties of complex networks has received a lot of attention [1-5]. Complex networks appear in such diverse disciplines as sociology, biology, chemistry, physics or computer science. In particular, great effort has been exerted to understand the behavior of technologically based communication networks such as the Internet [6], the World Wide Web [7], or e-mail networks [8-10]. However, the study of communication processes in a wider sense is also of interest in other fields, remarkably the design of organizations [11,12]. For instance, it is estimated that more than a half of the U.S. work force is dedicated to information processing, rather than to make or sell things in the narrow sense [11].

The pioneering work of Watts and Strogatz [1] opened a completely new field of research. Its main contribution was to show that many real-world networks have properties of random graphs and properties of regular low dimensional lattices. A model that could explain this observed behavior was missing and the proposed "small-world" model of the authors turned the interest of a large number of scientist in the statistical mechanics community in the direction of this appealing subject. Nevertheless, this simplified model gives rise to a connectivity distribution function with an exponential form, whereas many real world networks show a highly skewed degree distribution, usually with a power law tail

$$
P(k) \propto k^{-\gamma}
$$

with an exponent $2 \leq \gamma \leq 3$. Barabasi and Albert [2] proposed a model where nodes and links are added to the network in such a way that the probability of the added nodes to be linked to the old nodes depend on the number of existing connections of the old node. This simple computational model can explain the power law with an exponent $\gamma=3$.

Tools taken from statistical mechanics have been used to understand not only the topological properties of these communication networks, but also their dynamical properties. The main focus has been in the problem of searchability, although when the number of search problems that the network is trying to solve increases it raises the problem of congestion at some central nodes. It has 
been observed, both in real world networks [13] and in model communication networks [14-18], that the networks collapse when the load is above a certain threshold and the observed transition can be related to the appearance of the $1 / f$ spectrum of the fluctuations in Internet flow data $[19,20]$.

These two problems, search and congestion, that have so far been analyzed separately in the literature can be incorporated in the same communication model. In previous works $[16,21,18,22]$ we have introduced a collection of models that captures the essential features of communication processes and are able to handle these two important issues simultaneously. In these models, agents are nodes of a network and can interchange information packets along the network links. Each agent has a certain capability that decreases as the number of packets to deliver increases. The transition from a free phase to a congested phase has been studied for different network architectures in [16,18], whereas in [21] the cost of maintaining communication channels was considered. Finally in [22] we have attacked the problem of network optimization for fixed number of links and nodes.

This paper is organized as follows. In Sect. 2 we present well known results about search in complex networks, whereas in Sect. 3 we review recent work on network load, being considered as a betweenness centrality and hence a static characterization of the network. We present the common trends of our communication model in Sect. 4. In the next section, we show some of the exact results that have been obtained for a particular class of network, Cayley trees. Finally, in the last two sections we focus on the problem of network optimization, in the first one through a parameterized set of networks, including connectivities that can be short- or long-ranged, and different degrees of preferentiallity, and in the second one we perform an exhaustive search of optimal networks for a fixed number of nodes and links.

\section{Search in complex networks}

After the discovery of complex networks, one of the issues that has attracted a lot of attention is "search". Real complex communication networks such as the Internet or the World Wide Web are continuously changing and it is not possible to draw a map that allows to navigate in them. Rather, it is necessary to develop algorithms that efficiently search for the desired computers or the desired contents.

The origin of the study of this problem is in sociology since the seminal experiment of Travers and Milgram [23]. Surprisingly, it was found that the average length of acquaintance chains was about six. This means not only that short chains exist in social networks as reported, for example, in the "small world" paper by Watts and Strogatz [1], but even more striking that these short chains can be found using local strategies, that is without knowing exactly the whole structure of the social network.

The first attempt to understand theoretically the problem of searchability in complex networks was provided by Kleinberg [24]. In his work, Kleinberg 


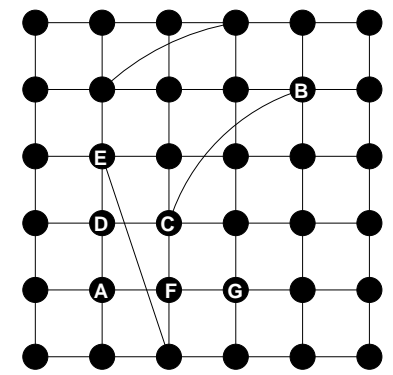

Fig. 1. Network topology and search in Kleinberg's scenario. Consider nodes $A$ and $B$. The distance between them is $\Delta_{A B}=6$ although the shortest path is only 3 . A search process to get from $A$ to $B$ would proceed as follows. From $A$, we would jump with equal probability to $D$ or $F$, since $\Delta_{D B}=\Delta_{F B}=5$ : suppose we choose $F$. The next jump would then be to $G$ or $C$ with equal probability since $\Delta_{C B}=\Delta_{G B}=4$, although from $C$ it is possible to jump directly to $B$. This is a consequence of the local knowledge of the network assumed by Kleinberg.

proposes a scenario where the network is modeled as a combination of a twodimensional regular lattice plus a number of long-range links. The distance $\Delta_{i j}$ between two nodes $i$ and $j$ is defined as the number of "lattice-steps" separating them in the regular lattice, that is disregarding long-range links (see Fig. 1). Long range links are not established at random. Instead, when a node $i$ establishes one of such links, it connects with higher probability with those nodes that are closer in terms of the distance $\Delta$. In particular, the probability that the link is established with node $j$ is

$$
\Pi_{i j} \propto\left(\Delta_{i j}\right)^{-r}
$$

where $r$ is a parameter.

The search algorithm proposed by Kleinberg is the following. A packet standing at one node will be sent to the neighbor of the node that is closer to the destination in terms of the distance $\Delta$. The algorithm is local because, as shown in Fig. 1, the heuristics of minimizing $\Delta$ does not warrant that the packet will follow the shortest path between its current position and its destination. Therefore, the underlying two-dimensional lattice has an imprecise global informational content.

Kleinberg showed that with this essentially local scenario (with imprecise global information), short paths cannot be found in general, unless the parameter $r$ is fixed to $r=2$. This raised the question of why real networks are then searchable, that is, how is it possible that in real networks local strategies are able to find paths that scale as $\log N$, where $N$ is the size of the network. Recently, Watts and coworkers have shown that with an idea similar to Kleinberg's, one can easily obtain searchable networks [25]. Their contribution consists in substituting the underlying low-dimensional lattice by an ultra-metric space where individuals are organized in a hierarchical fashion according to their preferences, similitudes, etc. In this case, a broad collection of networks turn out to be searchable. 
Parallel to these efforts, there have been some attempts to exploit the scale free nature of some networks to design algorithms that, being local in nature, are still quite efficient $[26,27]$. The idea in all these works is to profit from the scale-free nature of networks such as the Internet and bias the search towards those nodes that have a high connectivity and therefore act as hubs.

\section{Load and congestion in complex networks}

When the network has to tackle several simultaneous (or parallel) search problems it raises the important issue of congestion at overburdened nodes [13-17]. Indeed, for a single search problem the optimal network is clearly a highly centralized star-like structure, with one or various nodes in the center and all the rest connected to them. This structure is cheap to assemble in terms of number of links and efficient in terms of searchability, since the average cost (number of steps) to find a given node is always bounded ( 2 steps), independently of the size of the system. However, the star-like structure will become inefficient when many search processes coexist in parallel in the network, due to the limitation of the central node to process all the information.

Load, independently of search, has been analyzed in different classes of networks [28-31]. The load, as introduced in these works, is equivalent to the betweenness as it has been defined in social networks $[32,28]$. The betweenness of a node $j, \beta_{j}$, is defined as the number of minimum paths connecting pairs of nodes in the network that go through node $j$. Among the topological properties of networks, betweenness has become one of their main characteristics. In principle the time needed for the computation of the betweenness of all vertices is of order $\mathcal{O}\left(M N^{2}\right)$, where $N$ is the number of nodes and $M$ the number of links of the network. However, Newman [28] introduced an algorithm that reduces the magnitude of the time needed for the computation by a factor of $N$. This definition was used to measure the social role played by scientists in some collaboration networks [28]. Later on, it was also applied to quantify model networks. Thus, in [29] different networks are constructed and their distribution of betweennesses (or loads) measured. For instance, scale-free networks with an exponent $2<\gamma \leq 3$ lead to a load distribution which is also a power law, $P(\ell) \sim \ell^{-\delta}$ with $\delta \approx 2.2$. On the other side, the load distribution of small-world networks shows a combined behavior of two Poisson-type decays. In subsequent work, the authors in [31] suggested that real-world networks should be classified in two different universality classes, according to the exponent of the power-law distribution of loads. Finally, the distribution of loads was analytically computed for scale-free trees in [30].

The works discussed in the previous paragraph consider the betweenness as a topological property of the network, since it accounts for the number of shorter-paths going through a node. However, to take into account the search algorithm and the fact that packets can perform several random steps and then go through the same node more than once we introduce an effective betweenness. The effective betweenness of node $j, B_{j}$, represents the total number of packets 
that would pass through $j$ if one packet would be generated at each node at each time step with destination to any other node. The effective betweenness coincides with the topological betweenness when the nodes have complete information of the network structure and packets always follow the shortest paths between origin and destination.

\section{A model of communication}

The model that can handle search and congestion at the same time considers that the information is formed by discrete packets that are sent from an origin node to a destination node. Each node can store as many information packets as needed. However, the capacity of nodes to deliver information cannot be infinite. In other words, any realistic model of communication must consider that delivering, for instance, two information packets takes more time than delivering just one packet. A particular example of this would be to assume that nodes are able to deliver one (or any constant number) information packet per time step independently of their load, as happens in the communication model by Radner [11] and in simple models of computer queues [14,15,17], but note that many alternative situations are possible. In the present model, each node has a certain capability that decreases as the load of accumulated packets increases. This limitation in the capability of agents to deliver information can result in congestion of the network. Indeed, when the amount of information is too large, agents are not able to handle all the packets and some of them remain undelivered for extremely long periods of time. The maximum amount of information that a network can manage gives a measure of the quality of its organizational structure. In the study of the model, the interest is focused in both when the congestion occurs and how it occurs.

\subsection{Description of the model}

The dynamics of the model is as follows. At each time step $t$, an information packet is created at every node with probability $\rho$. Therefore $\rho$ is the control parameter: small values of $\rho$ correspond to low density of packets and high values of $\rho$ correspond to high density of packets. When a new packet is created, a destination node, different from the origin, is chosen randomly in the network. Thus, during the following time steps $t+1, t+2, \ldots, t+T$, the packet travels toward its destination. Once the packet reaches the destination node, it is delivered and disappears from the network. Another interpretation is possible for this information transfer scenario. Packets can be regarded as problems that arise at a certain ratio anywhere in an organization. When one of such problems arises, it must be solved by an arbitrary agent of the network. Thus, in subsequent time steps the problem flows toward its solution until it is actually solved. This problem solving scenario can be considered a particularly illustrative case of the more general information transfer scenario. The problem solving interpretation 
suggest a model similar to Garicano's [33] in that there is task diversity and agents are specialized in solving only certain types of tasks.

The time that a packet remains in the network is related not only to the distance between the source and the target nodes, but also to the amount of packets in its path. Indeed, nodes with high loads - i.e. high quantities of accumulated packets - will need long times to deliver the packets or, in other words, it will take long times for packets to cross regions of the network that are highly congested. In particular, at each time step, all the packets move from their current position, $i$, to the next node in their path, $j$, with a probability $q_{i j}$. This probability $q_{i j}$ is called the quality of the channel between $i$ and $j$, and is defined as

$$
q_{i j}=\sqrt{k_{i} k_{j}},
$$

where $k_{i}$ represents the capability of agent $i$ and, in general, changes with time. The quality of a channel is, thus, the geometric average of the capabilities of the two nodes involved, so that when one of the agents has capability 0 , the channel is disabled. It is assumed that $k_{i}$ depends only on the number of packets at node $i, \nu_{i}$, through:

$$
k_{i}=f\left(\nu_{i}\right)
$$

The function $f(n)$ determines how the capability evolves when the number of packets at a given node changes. In [18] we proposed a general form although in this paper we will only show results for the case in which the number of delivered packets is constant. This particular case is consistent with simple models of computer queues [14], although the precise definition of the models may differ from ours.

The election of the functional form for the quality of the channels and the capability of the nodes is arbitrary. Regarding the first, (3) is plausible for situations in which an effort is needed from both agents involved in the communi-

cation process. If, on the contrary, information can be transmitted without the collaboration of the receiver, an equation of the form

$$
q_{i j}=k_{i},
$$

would be more adequate. Equation (5) will be used for analytical understanding of the problem in Sect. 7, whereas (3) is used in Sect. 5. Some of the most relevant features of the model, however, are not dependent on which one is used.

\subsection{Congestion and network capacity}

Depending on the ratio of generation of packets $\rho$, two different behaviors are observed. When the amount of packets is small, the network is able to deliver all the packets that are generated and, after a transient, the total load $N$ of the network achieves a stationary state and fluctuates around a constant value. These fluctuations are indeed quite small. Conversely, when $\rho$ is large enough the number of generated packets is larger than the number of packets that the network can manage to solve and the network enters a state of congestion. Therefore, $N$ 


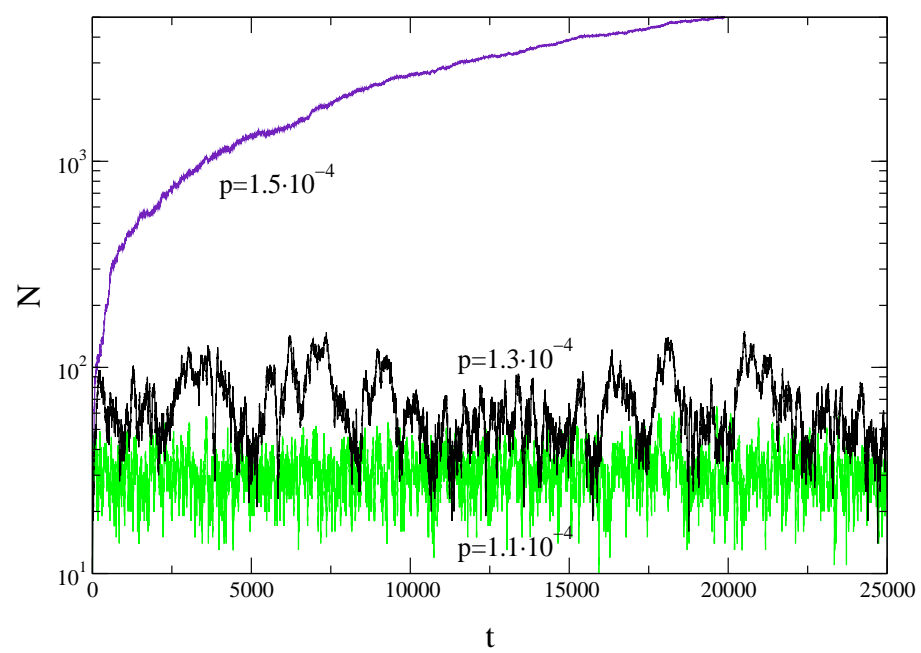

Fig. 2. Evolution of the total number of packets, $N$, as a function of time for a $(5,7)$ Cayley tree and different values of $\rho$, below the critical congestion point $\left(\rho=1.1 \cdot 10^{-4}<\right.$ $\left.\rho_{c}\right)$, above the critical congestion point $\left(\rho=1.5 \cdot 10^{-4}>\rho_{c}\right)$, and close to the critical congestion point $\left(\rho=1.3 \cdot 10^{-4} \approx \rho_{c}\right)$. Note the logarithmic scale in the $Y$ axis.

never reaches the stationary state but grows indefinitely in time. The transition from the free regime, $\rho$ small, to the congested regime, $\rho$ large, occurs for a well defined value of $\rho$, that will be denoted $\rho_{c}$. For values smaller than but close to $\rho_{c}$, the steady state is reached but large fluctuations arise.

The three behaviors (free, congested and close to the transition) are depicted in Fig. 2. For $\rho<\rho_{c}$, the width of the fluctuations is small, indicating short characteristic times. This means, among other thinks, that the average time required to deliver a packet to the destination is small. It also means that correlation times are short, that is, the state of the network at one time step has little influence on the state of the network only a few time steps latter. As $\rho$ approaches $\rho_{c}$, the fluctuations are wider and one can conclude that correlations become important. In other words, as one approaches $\rho_{c}$ the time needed to deliver a packet grows and the state of the network at one instant is determinant for its state many time steps later. In the congested regime, the amount of delivered packets is independent of the load and thus remains constant over time, while the number of generated packets is also constant, but larger than the amount of delivered packets. Thus, at each time step the number of accumulated packets is increased by a constant amount, and $N(t)$ grows linearly in time.

The transition from the free regime to the congested regime is therefore captured by the slope of $N(t)$ in the stationary state. When all the packets are delivered and there is no accumulation, the average slope is 0 while it is larger than 0 for $\rho>\rho_{c}$. We use this property to introduce an order parameter, $\eta$, that 


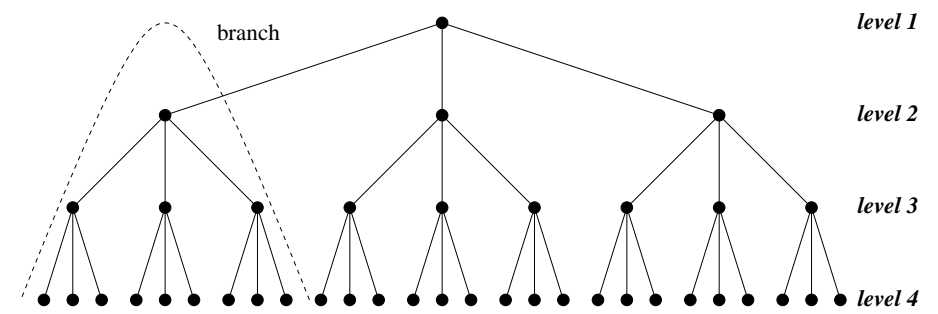

Fig. 3. Typical hierarchical tree structure used for simulations and calculations: in particular, it is a tree $(3,4)$. Dashed line: definition of branch, as used in some of the calculations.

is able to characterize the transition from one regime to the other:

$$
\eta(p)=\lim _{t \rightarrow \infty} \frac{1}{\rho S} \frac{\langle\Delta N\rangle}{\Delta t},
$$

In this equation $\Delta N=N(t+\Delta t)-N(t),\langle\ldots\rangle$ indicates an average over time windows of width $\Delta t$ and $S$ is the number of nodes in the system. Essentially, the order parameter represents the ratio between undelivered and generated packets calculated at long enough times such that $\Delta N \propto \Delta t$. Thus, $\eta$ is only a function of the probability of packet generation per node and time step, $\rho$. For $\rho>\rho_{c}$, the system collapses, $\langle\Delta N\rangle$ grows linearly with $\Delta t$ and thus $\eta$ is a function of $\rho$ only. For $\rho<\rho_{c},\langle\Delta N\rangle=0$ and $\eta=0$. Since the order parameter is continuous at $\rho_{c}$, the transition to congestion is a critical phenomenon and $\rho_{c}$ is a critical point as usually defined in statistical mechanics [34].

Once the transition is characterized, the first issue that deserves attention is the location of the transition point $\rho_{c}$ as a function of the parameters of the network. This transition point gives information about the capacity of a given network. Indeed, the maximum number of packets that a network can handle per time step will be $N_{c}=S \rho_{c}$. Therefore, $\rho_{c}$ is a measure of the amount of information an organization is able to handle and thus of the efficiency of a given organizational structure. One reasonable problem to propose is, therefore, which is the network that maximizes $\rho_{c}$ for a fixed set of available resources (agents and links).

\section{Analytical results for hierarchical lattices}

As a first step we considered hierarchical networks, since they provide a zeroth order approximation to real structures, and have also been used in the economics literature to model organizations $[11,35]$. In particular we are going to focus on hierarchical Cayley trees, as depicted in Fig. 3. Cayley trees are identified by their branching $z$ and their number of levels $m$, and will be denoted $(z, m)$ hereafter.

In this case the system is regarded as hierarchical also from a knowledge point of view. It is assumed in the model that agents have complete knowledge 
of the structure of the network in the subbranch they root. Therefore, when an agent receives a packet, he or she can evaluate whether the destination is to be found somewhere below. If so, the packet is sent in the right direction; otherwise, the agent sends the packet to his or her supervisor. Using this simple routing algorithm, the packets travel always following the shortest path between their origin and their destination.

As happens in other problems in statistical physics [36], the particular symmetry of the hierarchical tree allows an analytical estimation of the critical point $\rho_{c}$. In particular, the approach taken here is mean field in the sense that fluctuations are disregarded and only average expected values are considered. By using the steady state condition that the number of packets arriving at the top node, which is the most congested one, equals the number of packets leaving it we arrive to the following inequality

$$
\rho_{c} \geq \frac{\sqrt{z}}{\frac{z\left(z^{m-1}-1\right)^{2}}{z^{m}-1}+1}
$$

when the quality of the channels is given by (3). Although this expression provides an upper bound to $\rho_{c},(7)$ is an excellent approximation for $z \geq 3$, as shown in Fig. 4.

The total critical number of generated packets, $N_{c}=\rho_{c} S$, with $S$ denoting the size of the system, can be approximated, for large enough values of $z$ and $m$ such that $z^{m-1} \gg 1$, by

$$
N_{c}=\frac{z^{3 / 2}}{z-1}
$$

which is independent of the number of levels in the tree. It suggests that the behavior of the top node is only affected by the total number of packets arriving from each node of the second level, which is consistent with the mean field hypothesis.

According to (8), the total number of packets a network can deal with, $N_{c}$, is a monotonically increasing function of $z$, suggesting that, given the number of agents in the organization, $S$, the optimal organizational structure, understood as the structure with highest capacity to handle information, is the flattest one, with $m=2$ and $z=S-1$.

To understand this result it is necessary to take into account the following considerations:

- We are restricting our comparison only to different hierarchical networks and in any hierarchical network, the top node will receive most of the packets. Since origins and destinations are generated with uniform independent probabilities, roughly $(z-1) / z$ of the packets will pass through the top node.

- Still, it could seem that having small $z$ is slightly better according to the previous consideration. However, it is important to note that, in the present model (in particular due to (3)), the loads of both the sender and the receiver are important to have a good communication quality. In a network with small $z$, the nodes in the second level have also a high load, while in a network with a high $z$ the nodes in the second level are much less loaded. 

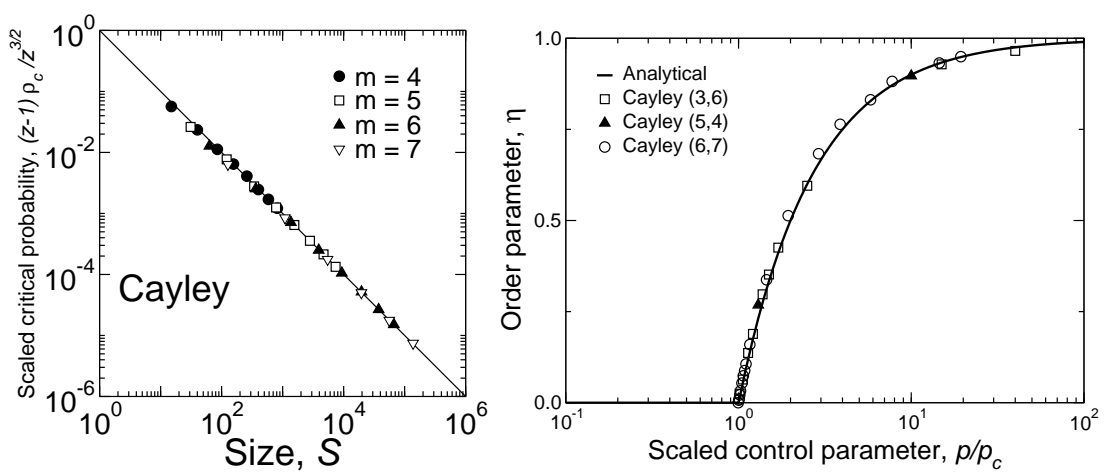

Fig. 4. Comparison between analytical (lines) and numerical (symbols) values obtained for hierarchical trees. Left: scaled critical probability (7). Right: order parameter (9).

- We have implicitly assumed that there is no cost for an agent to have a large amount of communication channels active.

For the order parameter, it is possible to derive an analytical expression for the simplest case where there are only two nodes that exchange packets. Since from symmetry considerations $\nu_{1}=\nu_{2}$, the average number of packets eliminated in one time step is 2 , while the number of generated packets is $2 \rho$. Thus $\rho_{c}=1$ and with the present formulation of the model it is not possible to reach the super-critical congested regime. However, $\rho$ can be extended to be the average number of generated packets per node at each step (instead of a probability) and in this case it can actually be as large as needed. As a result, the order parameter for the super-critical phase is $\eta=(\rho-1) / \rho$. As observed in Fig. 4, the general form

$$
\eta\left(\rho / \rho_{c}\right)=\frac{\rho / \rho_{c}-1}{\rho / \rho_{c}}
$$

fits very accurately the behavior of the order parameter for any Cayley tree.

\section{Optimization in model networks}

In this section we extend previous studies about local search in model networks in two directions. First, we consider networks that, as in Kleinberg's work, are embedded in a two-dimensional space, but study the effect not only of long range random links but also of long range preferential links. Secondly and more significantly, we consider the effect of congestion when multiple searches are carried out simultaneously. As we will show, this effect has drastic consequences for optimal network design.

\subsection{Network topology}

The small world model [1] considered two main components: local linking with neighbors and random long range links giving rise to short average distance be- 
tween nodes. The idea of Kleinberg is that local linking provides information about the social structure and can be exploited to heuristically direct the search process. Later, Barabasi and Albert showed that growth and preferential attachment play a fundamental role in the formation of many real networks [2]. Even though this model captures the correct mechanism for the emergence of highlyconnected nodes, it is not likely that it captures all mechanisms responsible for the evolution of "real-world" scale-free networks. In particular, it seems plausible that in many of the networks that show scale-free behavior there is also an underlying structure as in the Watts and Strogatz model. To illustrate this idea, consider web-pages in the World Wide Web. It is plausible to assume that a page devoted to physics is more likely to be connected to another page devoted to physics than to a page devoted to sociology. That is, a set of pages devoted to physics is likely to be more inter-connected than a set including pages devoted to physics and sociology.

Therefore we consider networks with four basic components: growth, preferential attachment, local attachment and random attachment. To create the network the following algorithm is used:

1. Nodes are located in a two-dimensional square lattice without interconnecting them.

2. A node $i$ is chosen at random.

3 . We create $m$ links starting at the selected node. With probability $\phi$, the destination node is selected preferentially. With probability $1-\phi$ the destination node is one of the nearest neighbors of the selected node. When the destination node is selected preferentially, we apply the following rule: the probability that a given destination node $j$ is chosen is a function of its connectivity

$$
\Pi_{j} \propto k_{j}^{\gamma}
$$

where $k_{j}$ is the number of links of node $j$ and $\gamma$ is a parameter that allows to tune the network from maximum preferentiallity to no preferentiallity. Indeed, for $\gamma=0$ the links are random and for $\gamma=1$ we recover the BA model, that generates scale free networks in the case $\phi=1$. For $\gamma>1$, a few nodes tend to accumulate all the links.

4. A new node is chosen and the process is repeated from step 3, until all the nodes have been chosen once.

Figure 5 shows two examples of networks in the process of being created according to this algorithm. Note that in this case, the number of links is fixed and the existence of long range links implies that some local links are not present and therefore that the information contained in the two-dimensional lattice is less precise.

\subsection{Communication model and search algorithm}

After the definition of the network creation algorithm, we move to the specification of the communication model and the search algorithm. For the communication model, we will use the general model presented and discussed in Sect. 


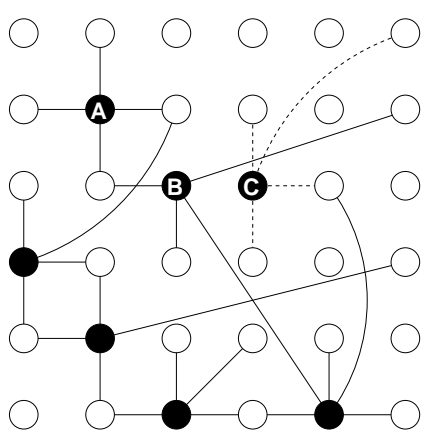

(a)

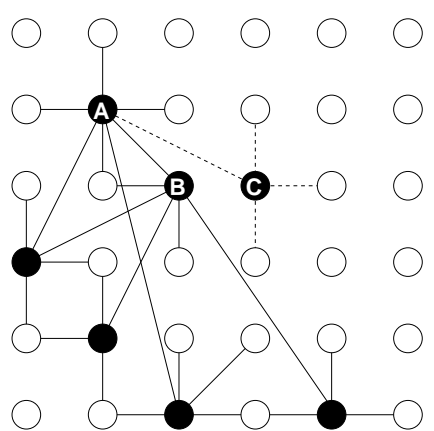

(b)

Fig. 5. Construction of networks with multiple linking mechanisms. In both cases $\phi=$ 0.25. A random node is selected at each time step and $m=4$ new links starting from that node are created. Black nodes represent nodes that have already been selected. Dotted lines represent the links created during the last time step in which node $C$ was selected. In (a), the destination of long range links is created at random $(\gamma=0)$, while in (b) they are created preferentially $(\gamma>0)$ and nodes $A$ and $B$ are attracting most of them.

4. As already stated, this model is general enough and considers the effect of congestion due to limitation of ability of nodes to handle information.

In comparison with hierarchical networks, there is only one ingredient of the communication model that needs to be reformulated. In the hierarchical version of the model, when a node receives a packet, it decides to send it downwards in the right direction if the solution is there, or upward to the agent overseeing her otherwise. This simple routing algorithm arises from the fact that we implicitly assume that the hierarchy is not only a communicational hierarchy, but also a knowledge hierarchy, where nodes know perfectly the structure of the network below them. In a complex network, this informational content of the hierarchy is lost. Here we will use Kleinberg's approach [24]. When an agent receives a packet, she knows the coordinates in the underlying two-dimensional space of its destination. Therefore, she forwards the packet to the neighbor that is closer to the destination according to the lattice distance $\Delta$ defined in Sect. 2, provided that the packet has not visited that node previously ${ }^{1}$. Note, however, that distance refers to the two-dimensional space, but not necessarily to the topology of the complex network and, as in Kleinberg's work, there might be shortcuts in directions that increase $\Delta$. Moreover, here long range links replace short range links and are not simply added to short range links. Therefore it is possible that following the direction of minimization of $\Delta$ the packet arrives to a dead end and has to go back.

Considering this algorithm, it is interesting that the three mechanisms to establish links (local, random and preferential) are somehow complementary. A completely regular lattice (all links are local) contains a lot of information since

\footnotetext{
${ }^{1}$ Packets are sent to previously visited nodes only if it is strictly necessary. This memory restriction avoids packets getting trapped in loops
} 
all the agents efficiently send their packets in the best possible direction. However, the average path length is extremely high in this networks and therefore the number of packets that are flowing in the network at a given time is also very high. The addition of random links can reduce dramatically the average path length, as in small world networks. However, if the number of random links is very high, then the number of local links is small and thus sending the packet to the node closer to the destination is probably quite inefficient (since it is possible that, even if it is very close in the underlying two-dimensional space, there is no short path in the actual topology of the network). Finally, preferential links seem to solve both problems. They obviously solve the long average path length problem but, in addition, the loss of information is not large, since the highly connected that actually concentrate this information. The star configuration is an extreme example of this: although there are no local links, the central node is capable of sending all the packets in the right directions. However, when the amount of information to handle is big, preferential links are especially inadequate because highly connected nodes act as centers of congestion. Therefore, optimal structures should be networks where all the mechanisms coexist: complex networks.

\subsection{Results}

We simulate the behavior of the communication model in networks built according to the algorithm presented in Sect. 4.1. First, a value of the probability of packet generation per node and time step, $\rho$, is fixed. For that particular value, we compare the performance of different networks: networks with different preferentiallity, from random $(\gamma=0)$ to maximum centralization $(\gamma \gg 1)$, and with different fraction of long range links, from pure regular lattices with no long range links $(\phi=0)$ to networks with no local component $(\phi=1)$. For each collection of the parameters $\rho, \gamma$, and $\phi$, the network load, $\bar{N}$, is calculated and averaged over a certain time window and over 100 realizations of the network, so that fluctuations due to particular simulations of the packet generation and of the network creation are minimized. As in the economics literature, the objective is to minimize the average time $\tau$ for a packet to go from the origin to the destination.

According to Little's Law of queuing theory [37], the characteristic time is proportional to the average total load, $\bar{N}$, of the network:

$$
\frac{\bar{N}}{\tau}=\rho S \Rightarrow \tau=\frac{\bar{N}}{\rho S}
$$

where $\rho$ is the probability of packet generation for each node at each time step. Thus, minimizing the average cost of a search is equivalent to minimizing the total load $\bar{N}$ of the network.

The main results are shown in Fig. 6. Consider first the behavior of the networks at low values of $\rho$. Figure 6 . a shows the load of the network for $\rho=0.01$ as a function of the fraction of long range links, $\phi$, both when they are random 


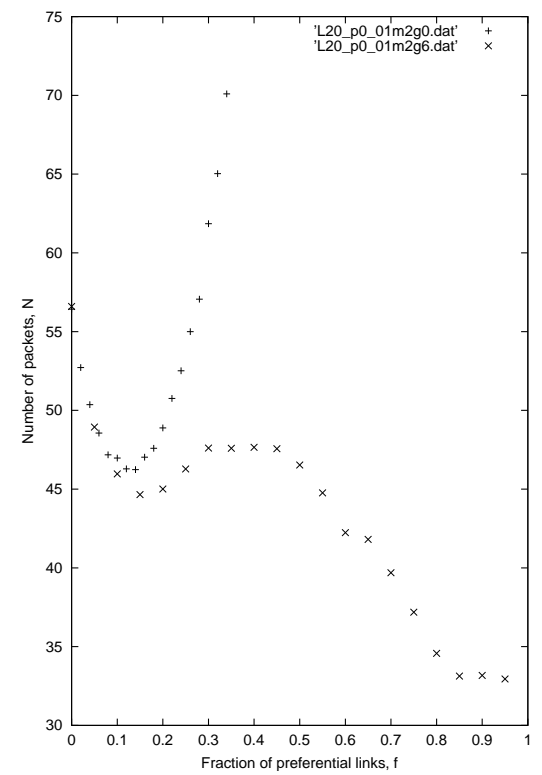

(a)

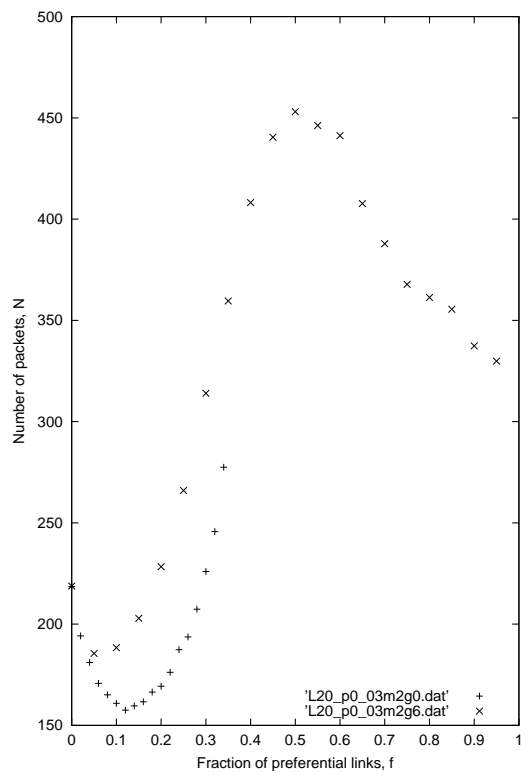

(b)

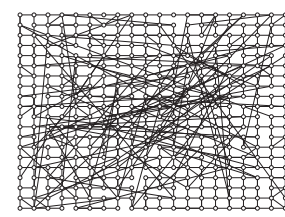

(c)

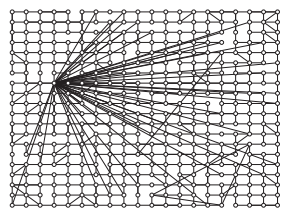

(d)

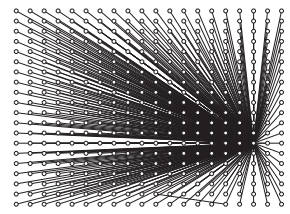

(e)

Fig. 6. (a) and (b) Average number of packets flowing in the network as a function of the fraction of preferential links: (a) $\rho=0.01$ and (b) $\rho=0.03$. Symbol $(+)$ corresponds to $\gamma=0$ (random links) and symbol $(\times)$ corresponds to $\gamma=6$ (extremely focused links). Figures (c),(d) and (e) show the typical shape of complex networks with particularly efficient configurations: (c) $\gamma=0$ and $\phi=0.12$; (d) $\gamma=6$ and $\phi=0.07$; and (e) $\gamma=6$ and $\phi=1.0$;

$\gamma=0$ and when they are extremely preferential $\gamma=6$. In the last case, long range links are established only with the most connected node. In this case of small $\rho$, centralization is not a big problem because congestion effects are still not important. Therefore, preferential links are, in general, better than random long range links. In the case of preferential links, it is interesting to understand the behavior of the curve $\bar{N}(\phi)$. For $\phi=0$ the network is a two-dimensional regular lattice and then the average distance between nodes is large. As some long range links are introduced, the average path length decreases as in the Watts-Strogatz model [1], and therefore the load of the network is smaller because packets reach their destination faster. However, the addition of long range links implies the lack 
of local links and when $\phi$ is further increased, the heuristic of minimizing the lattice distance $\Delta$ becomes worse and worse. This fact explains that for $\phi \approx 0.15$ (the network is similar to the one depicted in Fig. 6.d) the load has a local minimum that arises due to the trade-off between the two effects of introducing long range preferential links: shortening of the distances that tends to decrease $\bar{N}$ and destruction of the lattice structure that tends to decrease the utility of the heuristic search and then to increase $\bar{N}$. If $\phi$ is further increased, one node tends to concentrate all the links and for $\phi=1$ (Fig. 6.e) the network is strictly a star with one central node and the rest connected to it. In this completely centralized situation, the lack of two-dimensional lattice is not important because the packets will be sent to the central node and from there directly to the destination. Since for small $\rho$ congestion is not an issue, this structure turns out to be even better than the locally optimal structure with $\phi \approx 0.15$.

The situation is different when considering higher values of the probability of packet generation (Fig. 6.b displays the the results for $\rho=0.03$ ). Regarding preferential linking, the two locally optimal structures with $\phi=0.7$ and $\phi=1$ (Figs. 6.d and 6.e respectively) persist. However, in this situation and due to congestion considerations the first is better than the second. Thus, at some intermediate value of $0.01<\rho<0.03$, there is a transition such that the optimal structure changes from being the star configuration to being the mixed configuration with local as well as preferential links. Significantly, this transition is sharp, meaning that there is not a continuous pass from the star to the mixed.

Beyond the behavior of networks built with preferential long range links, it is worth noting that when the effect of the congestion is important (Fig. 6.b), the structure depicted in Fig. 6.c, where the long range links are actually thrown at random, becomes better than the structure in $6 . \mathrm{d}$. In other words, the optimal network is, in this case, a completely decentralized small world network a la Watts-Strogatz.

\section{Optimization in a general framework}

In the previous section we have compared the behavior of networks which have been built following different rules (nearest neighbor linking, preferential attachment, etc.). The main reason for focusing on a particular set of networks is that it is very costly to compare the performance of two networks: it is necessary to run a simulation, wait for the stationary state and calculate the average load of the network. Specially, close to the critical point the time needed to reach the stationary state diverges. In [22] we presented a formalism that is able to cope with search and congestion simultaneously, allowing the determination of optimal topologies. This formalism avoids the problem of simulating the dynamics of the communication process and provides a general scenario applicable to any communication process.

Let us focus on a single information packet at node $i$ whose destination is node $k$. The probability for the packet to go from $i$ to a new node $j$ in its next movement is $p_{i j}^{k}$. In particular, $p_{k j}^{k}=0 \forall j$ so that the packet is removed as soon 
as it arrives to its destination. This formulation is completely general, and the precise form of $p_{i j}^{k}$ will depend on the search algorithm and on the connectivity matrix of the network. In particular, when the search is Markovian, $p_{i j}^{k}$ does not depend on previous positions of the packet. In this case, the probability of going from $i$ to $j$ in $n$ steps is given by

$$
P_{i j}^{k}(n)=\sum_{l_{1}, l_{2}, \ldots, l_{n-1}} p_{i l_{1}}^{k} p_{l_{1} l_{2}}^{k} \cdots p_{l_{n-1} j}^{k} .
$$

This definition allows us to compute the average number of times, $b_{i j}^{k}$, that a packet generated at $i$ and with destination at $k$ passes through $j$.

$$
b^{k}=\sum_{n=1}^{\infty} P^{k}(n)=\sum_{n=1}^{\infty}\left(p^{k}\right)^{n}=\left(I-p^{k}\right)^{-1} p^{k} .
$$

and the effective betweenness of node $j, B_{j}$, is then defined as the sum over all possible origins and destinations of the packets,

$$
B_{j}=\sum_{i, k} b_{i j}^{k} .
$$

When the search algorithm is able to find the minimum paths between nodes, the effective betweenness will coincide with the topological betweenness, $\beta_{j}$, as usually defined $[32,28]$.

Once, these quantities have been defined, we focus on the load of the network, $N(t)$, which is the number of floating packets. These floating packets are stored in the nodes that act as queues. In a general scenario where packets are generated at random and independently at each node with a probability $\rho$, the arrival of packets to a given node $j$ is a Poisson process. In the original model presented in Sect. 4 we assumed that the quality of the channels depend on both the sender and the receiver nodes; if one assumes that it only depends on the receiver node then the delivery of packets is also a Poisson process. In this simple picture, the queues are called $M / M / 1$ in the computer science literature and the average load of the network is $[37,22]$

$$
\bar{N}=\sum_{j=1}^{S} \frac{\frac{\rho B_{j}}{S-1}}{1-\frac{\rho B_{j}}{S-1}} .
$$

There are two interesting limiting cases of equation (15). When $\rho$ is very small, taking into account that the sum of betweennesses is proportional to the average distance, one obtains that the load is proportional to the average effective distance. On the other hand, when $\rho$ approaches $\rho_{c}$ most of the load of the network comes from the most congested node, and therefore

$$
\bar{N} \approx \frac{1}{1-\frac{\rho B^{*}}{S-1}} \quad \rho \rightarrow \rho_{c},
$$

where $B^{*}$ is the effective betweenness of the most central node. The last results suggest the following interesting problem: to minimize the load of a network it 
is necessary to minimize the effective distance between nodes if the amount of packets is small, but it is necessary to minimize the largest effective betweenness of the network if the amount of packets is large. The first is accomplished by a star-like network, that is, a network with one central node and all the others connected to it. The second, however, is accomplished by a very decentralized network in which all the nodes support a similar load. This behavior is similar to any system of queues provided that the communication depends only on the sender.

It is worth noting that there are only two assumptions in the calculations above. The first one has already been mentioned: the movement of the packets needs to be Markovian to define the jump probability matrices $p^{k}$. Although this is not strictly true in real communication networks - where packets are not usually allowed to go through a given node more than once-it can be seen as a first approximation $[14,16,17]$. The second assumption is that the jump probabilities $p_{i j}^{k}$ do not depend on the congestion state of the network, although communication protocols sometimes try to avoid congested regions, and then $B_{j}=B_{j}(\rho)$. However, all the derivations above will still be true in a number of general situations, including situations in which the paths that the packets follow are unique, in which the routing tables are fixed, or situations in which the structure of the network is very homogeneous and thus the congestion of all the nodes is similar. Compared to situations in which packets avoid congested regions, it correspond to the worst case scenario and thus provide bounds to more realistic scenarios in which the search algorithm interactively avoids congestion.

Equation (15) relates a dynamical variable, the load, with the topological properties of the network and the properties of the algorithm. So we have converted a dynamical communication problem into a topological problem. Hence, the dynamical optimization procedure of finding the structure that gives the minimum load is reduced to a topological optimization procedure where the network is characterized completely by its effective betweenness distribution. In [22] we considered the problem of finding optimal structures for a purely local search, using a generalized simulated annealing (GSA) procedure, as described in $[38,39]$. On the one side, we have found that for $\rho \rightarrow 0$ the optimal network has a star-like centralized structure as expected, which corresponds to the minimization of the average effective distance between nodes. On the other extreme, for high values of $\rho$, the optimal structure has to minimize the maximum betweenness of the network; this is accomplished by creating a homogeneous network where all the nodes have essentially the same degree, betweenness, etc. One could expect that the transition centralized-decentralized occurs progressively. Surprisingly, the results of the optimization process reveal a completely different scenario. According to simulations, star-like configurations are optimal for $\rho<\rho^{*}$; at this point, the homogeneous networks that minimize $B^{*}$ become optimal. Therefore there are only two type of structures that can be optimal for a local search process: star-like networks for $\rho<\rho^{*}$ and homogeneous networks for $\rho>\rho^{*}$. 


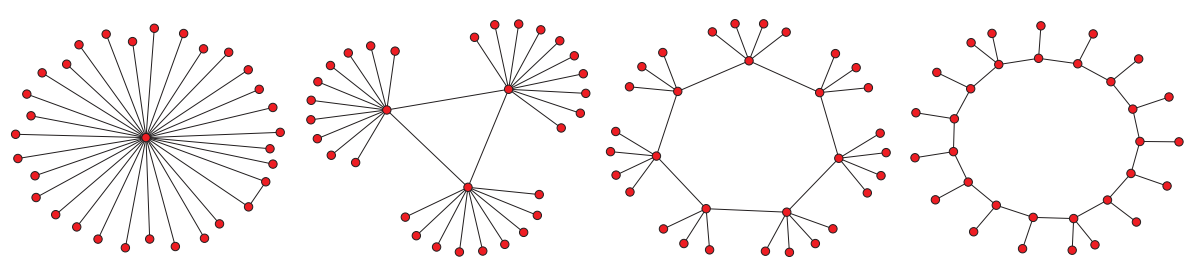

(a)

(b)

(c)

(d)

Fig. 7. Optimal topologies for networks with $S=32$ nodes, $L=32$ links and global knowledge. (a) $\rho=0.010$. (b) $\rho=0.020$. (c) $\rho=0.050$. (d) $\rho=0.080$. In this case of global knowledge, the transition from centralization to decentralization seems smooth.

Beyond the existence of both centralized and decentralized optimal networks, it is significant that the transition from one sort of networks to the other is abrupt, meaning that there are no intermediate optimal structures between total centralization and total decentralization. As already mentioned, this property is shared by the model networks in the previous section. Our explanation of this fact is the following. Since we are considering (in both the present and the last sections) local knowledge of the network topology, centered star-like configurations are extremely efficient in searching destinations and thus minimizing the effective distance between nodes. This explains that stars are optimal for a wide range of values of $\rho$, until the central node (or nodes) becomes congested. At this point, structures similar to stars will have the same problem and will be much worse regarding search; at this point, the only alternative is something completely decentralized, where the absence of congestion can compensate the dramatic increase in the effective distance between nodes. If this explanation is correct, one should be able to obtain a smooth transition from centralization to decentralization by considering global knowledge of the network, in such a way that the average effective distance (that in this case coincides with the average path length) is not much larger in an arbitrary network than in the star. Although we do not have extensive simulations in this case, Fig. 7 shows that there is some evidence to think that this is indeed the case.

\section{Summary}

We have presented some results concerning search and congestion in networks. By defining a communication model we have been able to cope with the problems of search and congestion simultaneously. For a hierarchical lattice some analytical results are found, by exploiting the symmetry properties of the network. For complex networks, this is not the case, and computational optimization to look for the best structures is required. On the one hand, for model networks where short-range, long-range, random and preferential connections are mixed we find that network that perform well for very low load become easily congested when the load is increased. On the other hand, when searching for optimal structures in a general scenario there is a clear transition from star-like centralized structures to homogeneous decentralized ones. 


\section{Acknowledgments}

This work has been supported by DGES of the Spanish Government, Grants No. PPQ2001-1519, No. BFM2000-0626, No. BEC2000-1029, and No. BEC20010980, and EC-FET Open Project No. IST-2001-33555.

\section{References}

1. D. Watts, S. Strogatz: Nature 393, 440 (1998)

2. A.-L. Barabasi, R. Albert: Science 286, 509 (1999)

3. L. A. N. Amaral, A. Scala, M. Barthelemy, H. E. Stanley: Proc. Nat. Acad. Sci. USA 97, 11149 (2000)

4. R. Albert, A.-L. Barabasi: Rev. Mod. Phys. 74, 47(2002)

5. S. Dorogovtsev, J. F. F. Mendes: Adv. Phys. 51, 1079 (2002)

6. M. Faloutsos, P. Faloutsos, C. Faloutsos: Comp. Comm. Rev. 29, 251 (1999)

7. R. Albert, H. Jeong, A.-L. Barabasi: Nature 401, 130 (1999)

8. M. E. J. Newman: Phys Rev E 66, 035101 (2002)

9. H. Ebel, L.-I. Mielsch, S. Bornholdt: Phys Rev E 66, 035103 (2002)

10. R. Guimera, L. Danon, A. Diaz-Guilera, F. Giralt, A. Arenas: cond-mat/0211498.

11. R. Radner: Econometrica 61, 1109 (1993)

12. S. DeCanio, W. Watkins: J. Econ. Behavior and Organization 36, 275 (1998)

13. V. Jacobson: 'Congestion avoidance and control'. In: Proceedings of SIGCOMM '88 (ACM, Standford, CA 1988)

14. T. Ohira, R. Sawatari: Phys. Rev. E 58, 193 (1998)

15. A. Tretyakov, H. Takayasu, M. Takayasu: Physica A 253, 315 (1998)

16. A. Arenas, A. Diaz-Guilera, R. Guimera: Phys. Rev. Lett. 86, 3196 (2001)

17. R. Sole, S. Valverde: Physica A 289, 595(2001)

18. R. Guimera, A. Arenas, A. Diaz-Guilera, F. Giralt: Phys. Rev. E 66, 026704 (2002)

19. I. Csabai: J. Phys. A: Math. Gen. 27, L417 (1994)

20. M. Takayasu, H. Takayasu, T. Sato: Physica A 233, 824(1996)

21. R. Guimera, A. Arenas, A. Diaz-Guilera: Physica A 299, 247 (2001)

22. R. Guimera, A. Diaz-Guilera, F. Vega-Redondo, A. Cabrales, A. Arenas: Phys. Rev. Lett. 89, 248701 (2002)

23. J. Travers, S. Milgram, Sociometry 32, 425 (1969)

24. J. Kleinberg: Nature 406, 845 (2000)

25. D. J. Watts, P. S. Dodds, M. E. J. Newman: Science 296, 1302 (2002)

26. L. A. Adamic, R. M. Lukose, A. R. Puniyani, B. A. Huberman: Phys. Rev. E 64, 046135 (2001)

27. B. Tadic: Eur. Phys. J. B 23, 221 (2001)

28. M. E. J. Newman: Phys. Rev. E 64, 016132 (2001)

29. K.-I. Goh, B. Kahng, D. Kim: Phys. Rev. Lett. 87, 278701 (2001)

30. G. Szabó, M. Alava, J. Kertész: Phys. Rev. E 66, 026101 (2002)

31. K.-I. Goh, E. Oh, H. Jeong, B. Kahng, D. Kim: Proc. Nat. Acad. Sci. USA 99, $12583(2002)$

32. L. C. Freeman, Sociometry 40, 35 (1977)

33. L. Garicano: J. Political Economy 108, 874 (2000)

34. H. E. Stanley: Introduction to phase transitions and critical phenomena (Oxford University Press, Oxford 1987).

35. P. Bolton, M. Dewatripont: Quart. J. Economics 109, 809 (1994) 
36. D. Stauffer, A. Aharony: Introduction to percolation theory, 2nd edn. (Taylor and Francis, 1992).

37. O. Allen: Probability, statistics and queueing theory with computer science application, 2nd edn. (Academic Press, New York 1990).

38. C. Tsallis, D. A. Stariolo: In Annual Review of Computational Physics II, ed. by D. Stauffer (World Scientific, Singapore 1994).

39. T. J. P. Penna: Phys. Rev. E 51, R1 (1995) 